\title{
Air-coupled Ultrasound Time Reversal (ACU-TR) for subwavelength non-destructive imaging
}

\author{
Torben Marhenke, Jürg Neuenschwander, Roman Furrer, Peter \\ Zolliker, Jens Twiefel, Jörg Hasener, Jörg Wallaschek, Sergio J. Sanabria Member, IEEE
}

\begin{abstract}
Air-coupled ultrasound (ACU) is increasingly used for non-destructive testing (NDT). With ACU, no contact or coupling agent (e.g., water, ultrasound gel) is needed between transducers and test sample, which provides high measurement reproducibility. However, for testing in production, a minimum separation is often necessary between sample and transducers to avoid contamination or transducer damage. Due to wave diffraction, the collimation of the ultrasound beam decreases for larger propagation distances, and ACU images become blurred and show lower defect lateral resolution with increasing sample-transducer separation. This is specially critical for thick composites, where large-size planar sources are used to bridge the large ACU transmission loss with good collimation. In this work, ACU re-radiation in unbounded media is extended to NDT of multi-layered composites. The extended method is named ACU time-reversal (ACU-TR), and significantly improves the defect resolution of ACU imaging. With ACU-TR, the complete pressure distribution radiated by large ACU source is measured with point receivers in one plane arbitrarily separated from the sample. By applying acoustic holography physics, it is then possible to quantitatively reconstruct the pressure field directly at arbitrary sample defect planes, which compensates for undesired diffraction phenomena and improves minimum detectable defect size, thereby achieving subwavelength lateral resolution. We tested the method on complex wood-based composite samples, based on ACU far-field measurements at a separation of 160 $\mathrm{mm}$ between sample and receiver transducer. With the proposed method, it is possible to detect surface defects as well as inner defects within composite boards. By using in the future point receiver arrays instead of a scanned microphone, both data $\sim \underset{F}{ }$ acquisition and evaluation can be potentially implemented in real time.
\end{abstract}

Index Terms - air-coupled ultrasound non-destructive testing, wood-based composite materials, gluing defects, inverse problem, time-reversal.

\section{INTRODUCTION}

T $\mathrm{N}$ recent years, the use of engineered composite materials has significantly increased as construction materials in lightweight construction, as reinforcement for highly stressed components, but also in the aerospace and sports industry

T. Marhenke was with the Institute of Dynamics and Vibration Research, Leibniz University Hannover, 30167 Hannover, Germany. He is now with - Fagus-GreCon Greten GmbH \& Co. KG, 31061 Alfeld, Germany (e-mail: Torben.Marhenke@gmail.com).

S. J. Sanabria is with the Institute of Diagnostic and Interventional Radiology, University Hospital Zurich, 8091 Zurich, Switzerland (e-mail: Sergio.Sanabria@usz.ch).

R. Furrer, Peter Zolliker and J. Neuenschwander are with the Swiss Federal Laboratories for Materials Science and Technology, Empa.

J.Twiefel and J.Wallaschek are with the Institute of Dynamics and Vibration Research, Leibniz University Hannover.

J. Hasener is with Fagus-GreCon Greten GmbH \& Co. KG, Alfeld.

Manuscript received XXX XX, 20XX; revised XXXX XX, 20XX.
[1]. These materials are composed of two or more bonded materials, which together show different material properties than the individual components. It is possible, for instance, to develop composites with direction-dependent material properties, which are specially adapted to the target application. Popular examples are fiber-plastic composites, wood-based materials, metal-matrix composites and reinforced concrete [2]. A main potential hazard of these composite materials are air inclusions and delamination during the bonding process, which can significantly reduce the composite strength and lead to structural failure. There is therefore high interest in developing non-destructive testing (NDT) methods to assess composite integrity in a non-invasive way, both during production and inservice.

Ultrasonic testing is a preferred method for bonding quality assessment, due to its high sensitivity to material discontinuities. Air inclusions are detected either as an increased reflection signal or as a reduced transmission signal [3]. Air-coupled ultrasound (ACU) testing has gained importance over the last decade, since the traditional influence of contact pressure and coupling medium (e.g., water, ultrasound gel)between ultrasound transducers and sample is eliminated. It is therefore possible to achieve reproducible measurements without sample contamination during on-going production. For these reasons, ACU is gradually becoming the standard method for non-destructive quality assurance of wood-based composites (ACU) [4]-[9]. ACU is also increasingly used in aerospace, civil engineering, food, textile, pharmacy and medical industries. ACU measurements are mainly carried out in a transmission setup [3], [10], [11]. Due to the additional acoustic impedance change as a result of the air layer resulting from the inclusions, the transmission signal drops at defect regions and the latter can be detected with high contrast [3], [7], [9], [12]. On the other hand, the large acoustic impedance mismatch between air and solids lead to high transmission loss $(<-50 \mathrm{~dB})$, and the acoustic attenuation in air increases above $200 \mathrm{kHz}$ exponentially with the square of the frequency [13]. Therefore, non-destructive testing at $\mathrm{MHz}$ frequencies are generally only feasible for composite plates of millimeter thickness, for which sufficient signal-to-noise ratio can be achieved. Focused transducers are typically used to improve the defect lateral resolution. [14]-[17]. For thicker composites, ACU transmission efficiency is optimized with large area transducers (diameter 10-50 times the wavelength size), and lower frequencies $(20-300 \mathrm{kHz})$ are utilized. In this case, planar non-focused transducers are used to achieve a planar collimated beam over a large field of view in depth. 


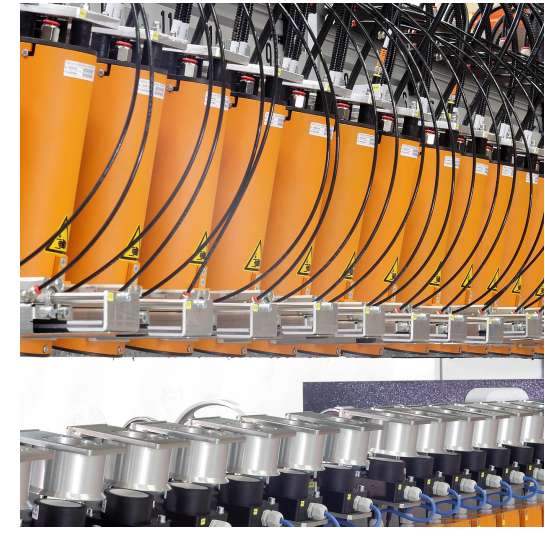

Fig. 1. Wood-based composite production line with ACU delamination detection channel-wise across the entire panel width [23].

However, this limits the lateral defect resolution to the size of the transducer elements [18]-[22].

Herein, ACU testing of wood-based composite panels during production is discussed as a target application. Wood-based materials have increasingly been used in contrast to solid wood [24], [25]. The most popular wood-based materials are particle boards (PB) or medium density fiberboards (MDF). During manufacture, the raw wood material is crushed into smaller elements, such as particles or fibers. After adding binders and adhesives (3 to $16 \%$ of the total mass, depending on the composite type [26]), the individual elements (mat) are bonded to the final product in a hot press. There is a risk of delamination during this process, which can be caused by the high moisture content of the mat, by short pressing times during the de-aeration phase, and sometimes due to insufficient curing of the binders before leaving the hot press [26]-[28]. These defects reduce the load-carrying capacity of the composite and can lead to structural failure. Recurring surface defects are a sign of damage or wrong adjustment of steel belts in the wood press. The location and distribution of surface delamination is key to characterize deviations in the pressing process and to plan press maintenance and repairs.

Bonding quality assessment is nowadays routinely performed with $\mathrm{ACU}$ in a pitch-catch transmission configuration, for which separate transmitter and receiver transducers are placed opposite sides of the sample. A photograph of a production line is shown in Fig.1. and the setup is described schematically in Fig.2a. An ultrasound beam is excited with normal incidence through the composite. The transmitter and receiver are aligned with respect to each other as a fixed unit. In production, multiple planar transmitter/receiver pairs provide transmission channels across the entire width of the panel. The composite is then scanned along its length to fully monitor production (Fig.1). Contrast images (C-scans) are calculated by measuring the peak waveform amplitude at each transmitter (TX) / receiver (RX) scan point. The transducers must be positioned at a minimum distance from the board to avoid damage due to the vapors escaping from the adhesives and the high mat temperatures (up to $100^{\circ} \mathrm{C}$ ) and to accommodate different board thicknesses [26]. The distance between transducers and sample limited is also limited in practice to certain a)

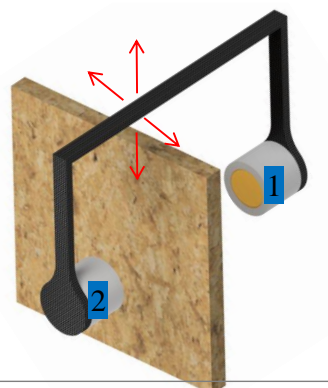

b)

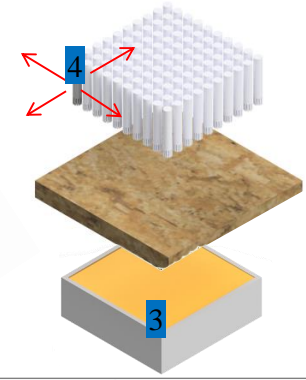

1: movable ACU transducer 3: fixed ACU transducer

2: movable ACU receiver $\quad 4$ : movable calibrated microphone

Fig. 2. ACU setup. a) Conventional pitch-catch setup. b) ACU Time Reversal (ACU-TR) setup proposed in this work.

distances by mechanical constraints, standardized system sizes and minimization of the effect of external acoustic interference sources. For example, in the production of particleboards, this distance is in the range of $160 \mathrm{~mm}$. Direct measurements of the ultrasound field at the sample surface are only possible with more involved techniques, such as laser vibrometry [29], [30].

The large transducer-sample distances are disadvantageous for defect lateral resolution. Using planar transducers, the ultrasound beam losses collimation beyond the near field length of the transmitter, and wave diffraction is observed around the defect edges. [31]-[33]. Focusing does not achieve a efficient transmission through centimeter-thick composites due to the reduced focal zone. In addition, economic aspects speak against it, since the small measurement area drastically increases the number of required channels to cover the full panel [6], [23]. In practice, planar transducers with a diameter of 20 to $70 \mathrm{~mm}$ are used to achieve sufficient ultrasound coupling, sample area coverage, and beam collimation for inspection of wood composite samples. Accurate adjustment of the transmitter-sample distance to the near field length is generally not possible due to the transducer positioning limitations described above and the varying panel thicknesses. The planar receiver measures an integrated pressure value over its entire surface, which leads to an additional reduction in resolution with increasing transducer size. As a rule of thumb, defects down to half the transducer diameter can be detected in wood composite samples with this setup. The wavelength $\lambda=c / f$ in the panel plane provides an additional resolution limit, and is a function of the speed of sound $c$ in the panel plane $(1500-2500 \mathrm{~m} / \mathrm{s})$ [8], [26] and the frequency $f$. Typically a maximum $f$ of $150 \mathrm{kHz}$ is usable to achieve efficient transmission through the panel with low attenuation in air and in the porous composite panel, and $\lambda \approx 15 \mathrm{~mm}$. Due to these general conditions, the reduction of defect resolution in wood-based materials represents a major challenge [3], [7], [34].

Several approaches have been proposed to post-process the ultrasound data and to improve the image resolution. Deconvolution algorithms aim at compensating the point spread function of the transducer, thus effectively compensating for 
the receiver averaging effect, and can reduce the blurring and improve the edge delineation in ultrasound images [7], [35]-[41]. However, deconvolution is typically applied to Cscans and does not take the wave phase information into account. Deconvolution filters are as well very sensitive to the transducer model and tend to amplify the noise in the images. Therefore, the effective defect resolution limit given by the smaller detectable defect size is not significantly improved. Recently, an ACU re-radiation method has been proposed to compute the volumetric pressure fields of real ACU transducers based on single plane measurements [42]. This work builds on the acoustic holography theory [43]-[45] and allows to compute the near field distribution of real ACU transducers from far field measurements.

In this work, we extend ACU re-radiation to NDT of composite panels. With this purpose, we acquire a far-field measurement of the pressure transmitted though the panel and reverse it in time to calculate the pressure fields directly at the composite defect planes. This allows compensating for diffraction effects and provide a major lateral resolution improvement in ACU NDT images. We denominate this method Air-coupled ultrasound Time-Reversal (ACU-TR). The new setup (Fig. 20) uses a fixed transmitter that covers the entire area to be examined. Then a point receiver array, here emulated with a scanning microphone, acquires the ultrasound signal transmitted through the panel. The setup is analogue to X-ray radiography, where a large parallel source generates radiation and a flat panel array receiver locally captures the incoming rays. We characterize the defect resolution of ACUTR based on well-defined defect geometries and compared to conventional pitch-catch transmission (Fig.2a) with and without deconvolution post-processing.

\section{THEORY}

\section{A. ACU Time Reversal (ACU-TR)}

Herein we derive the main building blocks of the ACUTR reconstruction. We base our derivations on [46]-[51]. The starting point is the acoustic wave equation for the description of the propagation of acoustic pressure $p$ field in linear, incompressible fluids as a function of the speed of sound $c$

$$
c^{2} \nabla^{2} p(\vec{x}, t)-\partial_{t}^{2} p(\vec{x}, t)=0,
$$

$p(\vec{x}, t)$ is the pulsed scalar pressure field in $3 \mathrm{D}$-space, where $\vec{x}=(x, y, z)$ and $t$ are space and time coordinates. $p(\vec{x}, t)$ is transformed in the Fourier domain into a superposition of harmonic place wave fields

$$
p(\vec{k}, \omega)=\int_{-\infty}^{\infty} \iiint_{-\infty}^{\infty} p(\vec{x}, t) \mathrm{e}^{-\mathrm{i}(\vec{k} \cdot \vec{x}+\omega t)} \mathrm{d} \vec{x} \mathrm{~d} t
$$

where $\omega$ is the harmonic frequency and $\vec{k}=\left(k_{x}, k_{y}, k_{z}\right)$ is the wave number vector. By similarly transforming Eq. (1) Helmholtz equation is obtained

$$
\nabla^{2} p+k^{2} p=0, k=\omega / c .
$$

Substituting Eq. (2) into Eq. (3) reduces one degree of freedom $k_{z}= \pm \sqrt{k^{2}-k_{x}^{2}-k_{y}^{2}}$. This allows building a holographic representation of Eq. 22, in which the volumetric pressure

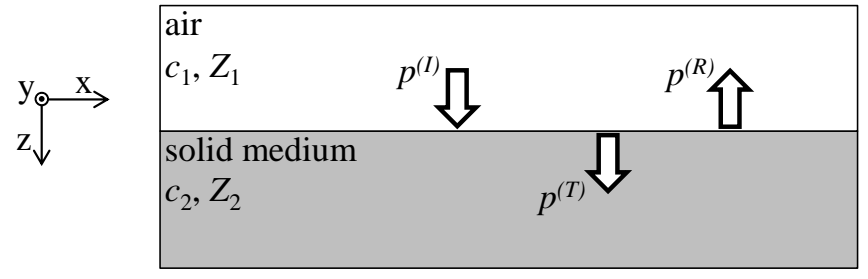

Fig. 3. Splitting of an incident pressure wave $p^{(I)}$ into a transmitted $p^{(T)}$ and reflected wave $p^{(R)}$ at a material interface.

field is calculated in function of a two-dimensional pressure measurement in an arbitrary plane $z=0$

$$
p\left(z, k_{x}, k_{y}, \omega\right)=p\left(0, k_{x}, k_{y}, \omega\right) \mathrm{e}^{-\mathrm{i} k_{z} z} .
$$

The positive root $k_{z}^{+}=\sqrt{k^{2}-k_{x}^{2}-k_{y}^{2}}$ describes an ultrasound wave forward propagating in an unbounded medium. For this case, an inverse transformation of Eq. (4) to the spatial domain can be calculated [47]

$$
p(\vec{x}, \omega)=\int_{-\infty}^{\infty} \int_{-\infty}^{\infty} p(\hat{x}, \hat{y}, 0, \omega) g_{\mathrm{PS}}(x-\hat{x}, y-\hat{y}, z) \mathrm{d} \hat{x} \mathrm{~d} \hat{y}
$$

where $g_{\mathrm{PS}}$ is a spherical source convolution kernel

$$
g_{\mathrm{PS}}(\vec{x})=\frac{\mathrm{e}^{-\mathrm{i} k\|\vec{x}\|_{2}^{2}}}{2 \pi\|\vec{x}\|_{2}^{2}}\left(\frac{1}{\|\vec{x}\|_{2}^{2}}+\mathrm{i} k\right) \frac{z}{\|\vec{x}\|_{2}^{2}} .
$$

Eq. (5) provides an holographic representation of the volumetric pressure field as a superposition of spherical waves distributed on an arbitrary plane $z=0$. This expression is a Rayleigh-Sommerfeld integral and an expression of Huygen's principle [51]. In ACU material testing, this method is extended to a multi-layered setup, in which wave reflection and refraction occur at the interfaces between air and solid media (Fig. 3). In the proposed forward setup, for each plane wave component an incident ultrasound wavefront $k_{z}^{+, 1}=\sqrt{k_{1}^{2}-k_{x}^{2}-k_{y}^{2}}$ propagates within a solid medium of speed of sound $c_{1}$. At the solid-air interface $(z=0)$, a reflected wave in the solid medium is generated, which propagates backwards and is described by a negative root of $k_{z}^{-, 1}=-\sqrt{k_{1}^{2}-k_{x}^{2}-k_{y}^{2}}$. Furthermore, a wave $k_{z}^{+, 2}=$ $\sqrt{k_{2}^{2}-k_{x}^{2}-k_{y}^{2}}$ is refracted across the solid-air interface. The latter diffracts in the air medium with speed of sound $c_{2}$ and is captured by the receiver microphone. For the two-layer case, Eq. (4) is re-written as:

$$
p(z)= \begin{cases}I p(0) \mathrm{e}^{-\mathrm{i} k_{z}^{+, 1} z}+R p(0) \mathrm{e}^{-\mathrm{i} k_{z}^{-, 1} z} & z<0 \\ T p(0) \mathrm{e}^{-\mathrm{i} k_{z}^{+, 2} z} & z>0 .\end{cases}
$$

where $\left(k_{x}, k_{x}, \omega\right)$ terms are herein dropped from $p(z), p(0), I$ and $R$ to shorten notation. $I$ describes the pressure amplitude of the incident wave in the first medium, $R$ the reflected amplitude, and $T$ the transmitted amplitude into the second medium. $I=1$ is an arbitrary excitation pressure, and $R$ and $T$ are calculated by applying two boundary conditions at the interface. In particular the pressure $p$ is continuous at $z=0$, as well as the normal particle displacement $u_{x}=c \omega^{2} Z^{-1} \partial_{z} p(z)$, 
a)

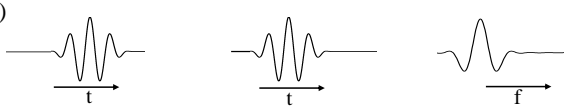

1.Time data $\mathrm{p}(\mathrm{x}, \mathrm{y}, \mathrm{t}) \rightarrow 2$.Time mirror $\mathrm{p}(\mathrm{x}, \mathrm{y}, \mathrm{t}) \rightarrow 3$.DFT $\mathrm{p}(\mathrm{x}, \mathrm{y}, \mathrm{f})$

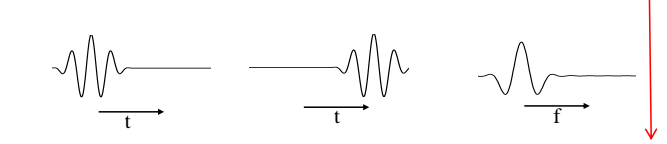

6.Time mirror $\mathrm{p}(\mathrm{x}, \mathrm{y}, \mathrm{z}, \mathrm{t})<5$.IDFT $\mathrm{p}(\mathrm{x}, \mathrm{y}, \mathrm{z}, \mathrm{-t}) \leftarrow 4$.Re-rad. $\mathrm{p}(\mathrm{x}, \mathrm{y}, \mathrm{z}, \mathrm{f})$

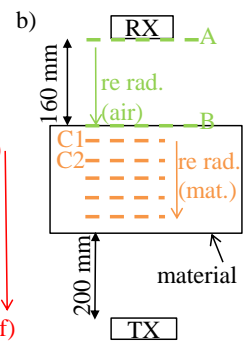

Fig. 4. ACU time-reversal. a) Computation steps. b) General geometric setup. From an arbitrary plane A we compute the pressure field at the sample surface (B) or arbitrary defect planes within the composite material $(\mathrm{C} 1, \mathrm{C} 2$ ...). Also mark $\mathrm{z}=160 \mathrm{~mm}$ in $\mathrm{b}$ ) to keep coherence with a). Finally, you should switch a) and b) to keep the same referencing order as in the text (and update the corresponding Fig. references).

which results in

$$
T=2\left(1+\frac{Z_{1} c_{2} k_{z}^{+, 2}}{Z_{2} c_{1} k_{z}^{+, 1}}\right)^{-1}
$$

and $R=T-1$, where $Z_{1}, Z_{2}$ are the acoustic impedance of both media. The so far calculated expressions describe the forward re-radiation problem, that is, the calculation of diffracted pressure fields $p$ away from the source plane. For ACU-TR the inverse problem is solved to back-project the measured fields at the receiver plane (A) to closer locations to the transmitter source corresponding to the panel surface (B) or different defect planes $(\mathrm{C} 1, \mathrm{C} 2)$ within (Fig. $4 \mathrm{~b})$. With this purpose, the time reversibility of the wave equation is used [52]. Eq. [1] is satisfied by time-reversed solutions $p(\vec{x},-t)$, which describe back-propagation and virtual focusing of the pressure fields towards the originating source. In the Fourier domain, these solutions are equivalent to a mirrored frequency spectrum $p(\vec{x},-t) \mapsto p(\vec{x},-\omega)$. For ACU-TR calculation in multi-layered media, Eq. (7) is used. In this case, the transmitted wave $k_{z}^{+, 2}$ measured by the microphone is timereversed to calculate the incident wave $k_{z}^{+, 1}$ in the solid medium. Consequently, the transmitted amplitude $T=1$ is known from the microphone measurement and the incident amplitude $I$ is calculated with the above boundary conditions

$$
I=\frac{1}{2}\left(1+\frac{Z_{1} c_{2} \sqrt{k_{2}^{2}-k_{x}^{2}-k_{y}^{2}}}{Z_{2} c_{1} \sqrt{k_{1}^{2}-k_{x}^{2}-k_{y}^{2}}}\right) .
$$

\section{B. Practical implementation of $A C U-T R$}

The practical implementation of ACU-TR for defect imaging is illustrated in Fig. $4 \mathrm{p}$. A pressure field measured by a point receiver microphone in an arbitrary plane $p_{A}(x, y, t)$, which is parallel to the sample surface, is time-reversed and re-radiated in air until the sample surface $p_{B}(x, y, t)$. For defect imaging within the sample the pressure field $p_{B}(x, y, t)$ is re-radiated again to an arbitrary defect imaging plane $p_{C}(x, y, z, t)$ within the sample.

The Rayleigh-Sommerfeld integral Eq. (5) is used to describe the re-radiation process in air. In numerical computation, Eq. 57 is discretized as a summation of elementary circular point sources within a two-dimensional measurement window of size $N$ and pixel size $h$

$$
p(\vec{x}, \omega) \cong \sum_{s=1}^{N} h^{2} p\left(\hat{x}_{s}, \hat{y}_{s}, 0, \omega\right) g_{\mathrm{PS}}\left(x-\hat{x}_{s}, y-\hat{y}_{s}, z\right),
$$

where $\hat{x}_{s}$ and $\hat{y}_{s}$ are the coordinates of the sources within the measurement plane. If measurements $\left(\hat{x}_{s}, \hat{y}_{s}\right)$ are available over a discrete grid with uniform step $h$, two-dimensional (2D) Fourier transforms can be used to speed up numerical calculations of field propagation from one plane to another for pressure fields in the same way as electrical fields. Hack [53] and Zolliker [54] for example applied such a method for holographic Terahertz imaging. The theoretical basics are given by Delen et al. [55] for electric field propagation between two planes. Similarly, the integral Eq. (5) is in the form of a two-function convolution, which can be numerically calculated as a product of 2D Discrete Fourier Transforms $\mathcal{F}_{2 D}:\left(\hat{x}_{s}, \hat{y}_{s}\right) \mapsto\left(k_{x}, k_{y}\right)$

$p\left(\hat{x}_{s}, \hat{y}_{s}, z\right)=\mathcal{F}_{2 D}^{-1}\left\{\mathcal{F}_{2 D}\left\{p\left(\hat{x}_{s}, \hat{y}_{s}, 0\right)\right\} \mathcal{F}_{2 D}\left\{g_{P S}\left(\hat{x}_{s}, \hat{y}_{s}, z\right)\right\}\right\}$.

For multi-layered media, the 2D Fourier Domain representation also allows numerical calculation of the effect of the interface based on Eq. (7) and Eq. (9)

$p_{C}\left(\hat{x}_{s}, \hat{y}_{s}, z\right)=\mathcal{F}_{2 D}^{-1}\left\{\mathcal{F}_{2 D}\left\{p_{B}\left(\hat{x}_{s}, \hat{y}_{s}, 0\right)\right\} I\left(k_{x}, k_{y}\right) \mathrm{e}^{-\mathrm{i} k_{z}^{+, 1} z}\right\}$.

$p_{B}\left(\hat{x}_{s}, \hat{y}_{s}, 0\right)$ is the pressure field at the sample interface on the receiver side, which is calculated from the microphone measurement $p_{A}\left(\hat{x}_{s}, \hat{y}_{s}, 0\right)$ with Eq. 10) or Eq. 111. $p_{C}\left(\hat{x}_{s}, \hat{y}_{s}, z\right)$ is the pressure calculated within the sample at arbitrary depth $z$ from its surface.

The time waveforms at every location of space are then calculated from Eq. (10), (11) or (12) with a one-dimensional (1D) Inverse Discrete Fourier Transform $\mathcal{F}_{1 D}^{-1}: \omega \mapsto t[56]$

$$
p(\vec{x}, t)=F_{1 D}^{-1}\{p(\vec{x}, \omega)\} .
$$

Similarly, the 1D Discrete Fourier Transform $\mathcal{F}_{1 D}: t \mapsto \omega$ is used to transform the measured fields $p\left(\hat{x}_{s}, \hat{y}_{s}, 0, t\right)$ to the Fourier domain $p\left(\hat{x}_{s}, \hat{y}_{s}, 0, \omega\right)$.

In order to achieve virtual focusing and back-propagate the measured pressure fields towards the originating source, time-reversal is applied to the measured fields. The steps are summarised in Fig. 4 and have been explained in detail in [42] and [57] for calculating the near-field pressure field at the surface of a transducer from pressure measurements in an arbitrary far-field plane. For calculating time-reversed waveforms, the measured field $p(x, y, 0, t)$ is mirrored in time $p(x, y, 0,-t)$ prior to 1D Fourier transformation and reradiation is calculated with Eq. (10, , 11), 12. The resulting fields $p(x, y, z,-t)$ are again mirrored in the time-domain after the inverse 1D Fourier transformation Eq. (13). An alternative to this procedure is to calculate the Fourier Transform of $p(x, y, 0, t)$ instead and change the sign of $k$ in the re-radiation equations Eq. (10), (11), (12). 


\section{Deconvolution}

The above described ACU-TR method uses time-dependent pressure data, thus utilizing both phase and amplitude information. A deconvolution algorithm, which is based on postprocessing of ACU amplitude images is here outlined for comparison with ACU-TR [58].

Deconvolution is applied as post-processing of conventional ACU C-scan images generated with the pitch-catch setup (Fig.22. At each scanned pixel $(x, y)$, the smearing introduced by the pressure field distribution excited by the finite-size ACU transmitter and the integration pressure field over the finite ACU receiver area are both approximated with the point spread functions $h_{\mathrm{TX}}(x, y)$ and $h_{\mathrm{RX}}(x, y)$, respectively

$$
v(x, y)=\iint_{-\infty}^{\infty} t(x-\hat{x}, y-\hat{y}) h_{\mathrm{RX}}(\hat{x}, \hat{y}) h_{\mathrm{TX}}(\hat{x}, \hat{y}) \mathrm{d} \hat{x} \mathrm{~d} \hat{y}
$$

where $t(x, y)$ represents the transmission coefficient (amplitude contrast) at the defect plane in linear scale. By grouping $h_{\mathrm{TX}}(x, y)$ and $h_{\mathrm{RX}}(x, y)$ in a single term $h(x, y)=$ $h_{\mathrm{TX}}(x, y) h_{\mathrm{RX}}(x, y)$, Eq. (14) can be re-written as a two dimensional convolution $v(x, y)=t(x, y) *_{2 \mathrm{D}} h(x, y)$, which is equivalent in the Fourier domain to the product $V\left(\omega_{\mathrm{x}}, \omega_{\mathrm{y}}\right)=$ $T\left(\omega_{\mathrm{x}}, \omega_{\mathrm{y}}\right) H\left(\omega_{\mathrm{x}}, \omega_{\mathrm{y}}\right)$ [59], where $\omega_{\mathrm{x}}=2 \pi f_{x}$ and $\omega_{\mathrm{y}}=2 \pi f_{y}$ are spatial frequencies. The two-dimensional deconvolution filter $D\left(\omega_{\mathrm{x}}, \omega_{\mathrm{y}}\right)$ compensates the transducer smearing $H\left(\omega_{\mathrm{x}}, \omega_{\mathrm{y}}\right)$ with an inverse estimate $1 / \tilde{H}\left(\omega_{\mathrm{x}}, \omega_{\mathrm{y}}\right)$, from which an estimation of the transmission coefficient at the defect plane is obtained:

$$
\tilde{T}\left(\omega_{\mathrm{x}}, \omega_{\mathrm{y}}\right)=\tilde{V}\left(\omega_{\mathrm{x}}, \omega_{\mathrm{y}}\right) \tilde{D}\left(\omega_{\mathrm{x}}, \omega_{\mathrm{y}}\right) .
$$

Deconvolution is inherently ill-conditioned for $\mathbf{H} \rightarrow 0$, where D leads to noise amplification. To alleviate this effect, a Wiener regularization term is used to attenuate all frequency components of $\tilde{\mathbf{H}}$ with an amplitude below a fraction $\kappa$ of the maximum $\tilde{\mathbf{H}}$ value. Both $\kappa$ and the order of the Wiener filter s are tuning parameters that are manually adjusted to achieve an optimum trade-off between resolution improvement and noise amplification [60]-[63]. For the setup of Fig.2, $h(x, y)$ is approximated by a circular window of radius $a, \tilde{h}(x, y)=$ $\operatorname{rect}\left(\frac{\sqrt{x^{2}+y^{2}}}{2 a}\right)$, for which $\tilde{H}\left(\omega_{\mathrm{r}}\right)=\frac{2 \pi a}{\omega_{\mathrm{r}}} J_{1}\left(\omega_{\mathrm{r}} a\right)$ [59]. For $\omega_{\mathrm{r}}<1,8826 / a$ (intersection), $\tilde{H}$ accurately converges to a damped sinusoidal function $\tilde{H}\left(\omega_{\mathrm{r}}\right)=\frac{\sqrt{8 a \pi}}{\omega_{\mathrm{r}}^{3}} \cos \left(a \omega_{\mathrm{r}}-\frac{3 \pi}{4}\right)$. The $\pi \mathrm{rad}$ phase shifts at zero-crossings of $\tilde{H}$ led to an ill-conditioned deconvolution. By removing the phase information from $\tilde{H}$, a smooth positive monotonically decreasing function $\tilde{H}$ is obtained. The resulting filter is written as

$$
D\left(\omega_{\mathrm{r}}\right)=\frac{1}{\tilde{H}\left(\omega_{\mathrm{r}}\right)} \frac{1}{1+\left(\frac{\eta \max _{\omega_{\mathrm{r}}}\left|\tilde{H}\left(\omega_{\mathrm{r}}\right)\right|}{\left|\tilde{H}\left(\omega_{\mathrm{r}}\right)\right|}\right)^{s}},
$$

with

$$
\tilde{H}\left(\omega_{\mathrm{r}}\right)= \begin{cases}\pi a^{2} & \omega_{\mathrm{r}}=0 \\ \frac{2 \pi a}{\omega_{\mathrm{r}}} J_{1}\left(\omega_{\mathrm{r}} a\right) & \omega_{\mathrm{r}} \leq \frac{1,8826}{a} \\ \sqrt{\frac{8 a \pi}{\omega_{\mathrm{r}}^{3}}} & \omega_{\mathrm{r}}>\frac{1,8826}{a}\end{cases}
$$

For implementation, DFTs with sampling frequency $\omega_{\mathrm{s}=2 \pi / \Delta_{f}}$

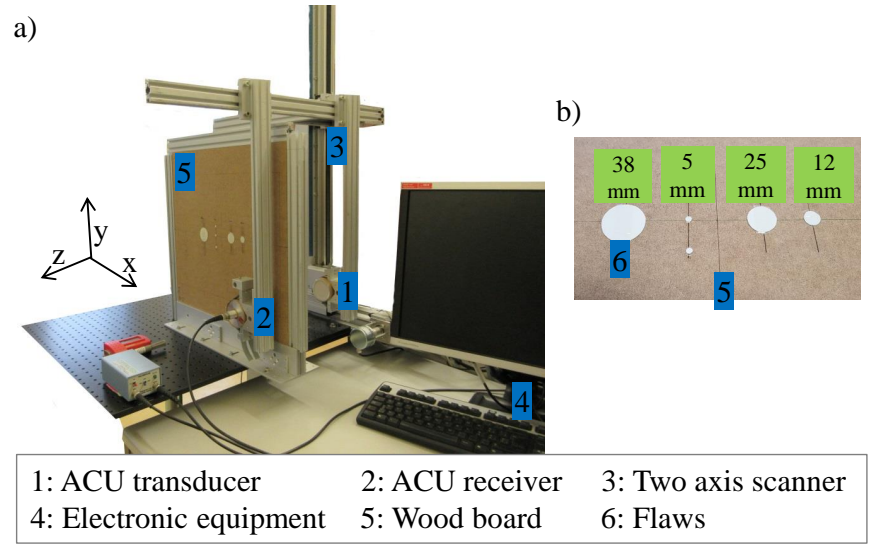

Fig. 5. ACU pitch-catch setup. a) Measurement arrangement. b) Simulated flaws.

were used, with resolution determined by the scanning step $\Delta_{f}$, which led to negligible aliasing artifacts $(<45 \mathrm{~dB})$. A raised-cosine apodization window was introduced around the image boundaries in order to minimize overshot (Gibbs artifacts) due to amplitude discontinuities at the edges of the image.

\section{EXPERIMENTS}

A Medium Density Fiberboard (MDF) and a Particleboard (PB) were used as sample wood-based composites for the investigations. These are anisotropic, biological materials, so that the transmitted pressure fields in air are highly heterogeneous. Therefore, it is a good use case to show the generality of the ACU-TR method. In particular, a MDF panel with a thickness of $22 \mathrm{~mm}$ and an average density of 713 $\mathrm{kg} / \mathrm{m}^{3}$, and a PB panel with a thickness of $19 \mathrm{~mm}$ and an average density of $639 \mathrm{~kg} / \mathrm{m}^{3}$ were tested. To simulate delamination, paper shreds with diameters of 5, 12, 25 and 38 $\mathrm{mm}$ were positioned on top of the board (see Fig. 5b), since an artificial creation of delaminations with well-controlled size is technically challenging due to the uncontrolled spread of the adhesive during board pressing. Due to the similar material parameters of paper and wood, delaminations can be simulated realistically, as shown by previous work [33].

\section{A. Conventional ACU pitch-catch setup}

Two ACU planar transducers were aligned perpendicularly to the surfaces of the sample, one transmitting an ultrasonic signal and the other one receiving it. The TX/RX pair I has a central frequency of $120 \mathrm{kHz}$ and a $50 \mathrm{~mm}$ active diameter (NCG100-D50, The Ultran Group Inc., State College, PA, USA). Both the transmitter TX and the receiver RX were attached to a $\mathrm{C}$-arm, so that there was no relative displacement between TX and RX. The C-arm was bolted to a scanner that allowed the entire panel to be inspected (see Fig.5 5 ). The scanning step was $1 \mathrm{~mm}$ and the positioning reproducibility of the scanning system is $\pm 0.02 \mathrm{~mm}$. The distance between TX and sample was adjusted to the near field distance $(220 \mathrm{~mm})$, where a natural focusing of the transmitter is observed [51]. For the MDF board, Rx measurement planes were acquired both at $160 \mathrm{~mm}$ and at $5 \mathrm{~mm}$ above the board (Fig. 7). 


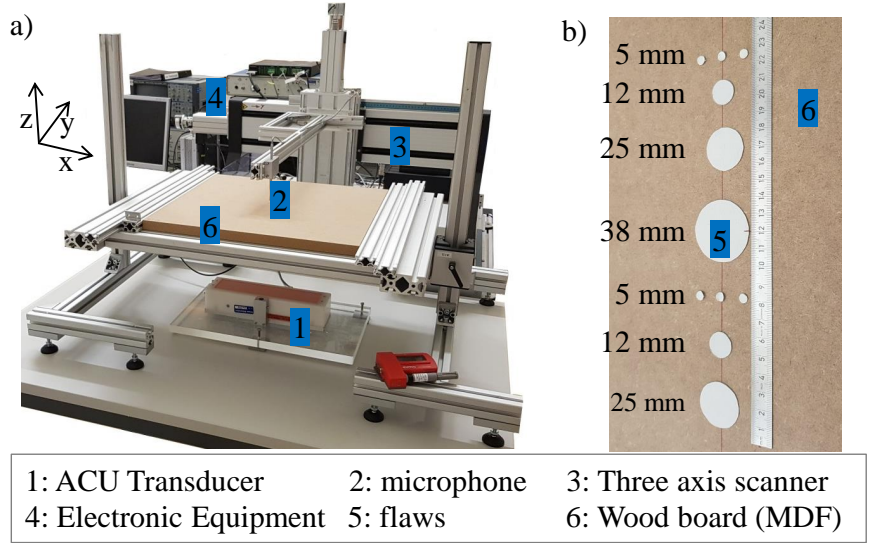

Fig. 6. ACU Time Reversal (TR) setup. a) Measurement arrangement. b) Simulated flaws.

\section{B. ACU Time Reversal (ACU-TR)}

For the imaging of delamination defects with ACU-TR, a larger rectangular $(50 \mathrm{~mm} \times 250 \mathrm{~mm})$ transducer (TX II) with an operating frequency of $120 \mathrm{kHz}$ (NCI100-50x250, Ultran) was used (see Fig. 6). The large size of the transducer allowed examination of several defects at the same time without scanning the transmitter (TX II), thus providing a full-field measurement. The distance between TX and sample was the same as in the conventional ACU pitch-catch setup $(220 \mathrm{~mm})$.

A calibrated pressure-field microphone (RX II) (4138, Brel \& Kjr, Nrum, Denmark), with a sensitivity of $10 \mathrm{mV} / \mathrm{Pa}$ and a polarization voltage of $200 \mathrm{~V}$ was used to measure the sound pressure. This microphone was mounted on the three-dimensional scanner to measure the pressure at defined positions. The spatial step of ACU-TR scans depended on the wavelength in air. According to [42], [64], to avoid discretization artifacts the scanning resolution should be roughly less than one third of the wavelength. Therefore, for ACU-TR, the calculated scanning resolution $\Delta_{s}$ was

$$
\Delta_{s}=\frac{\lambda}{3}=\frac{c}{3 f}=\frac{344 \mathrm{~m} / \mathrm{s}}{3 \cdot 120000 \mathrm{~Hz}} \cong 1 \mathrm{~mm},
$$

where $c_{2}=344 \mathrm{~m} / \mathrm{s}$ is the nominal speed-of-sound in air [65]. The scanning area of the receiver was rectangular, and its boundaries were defined by a $30 \mathrm{~dB}$ decrease in relation to the maximum pressure. This has been previously shown to be an adequate cut-off value to capture most of the ACU field features [42].

For the MDF board, Rx measurement planes were acquired both $160 \mathrm{~mm}$ (Fig. 8) and $5 \mathrm{~mm}$ (Fig. 9) above the board. The latter were directly used to validate the ACU-TR calculations (Fig. 9, 10 and 11). The defects were positioned on the sample surface on the receiver Rx side.

For the PB sample, measurements were acquired with the receiver RX located $160 \mathrm{~mm}$ over the board. In this case, measurements were successively performed with defects on either receiver side (Fig. 12) or transmitter side (Fig. 13). The latter case serves as a general demonstration of re-radiation through the full thickness of the wood composite panel. This

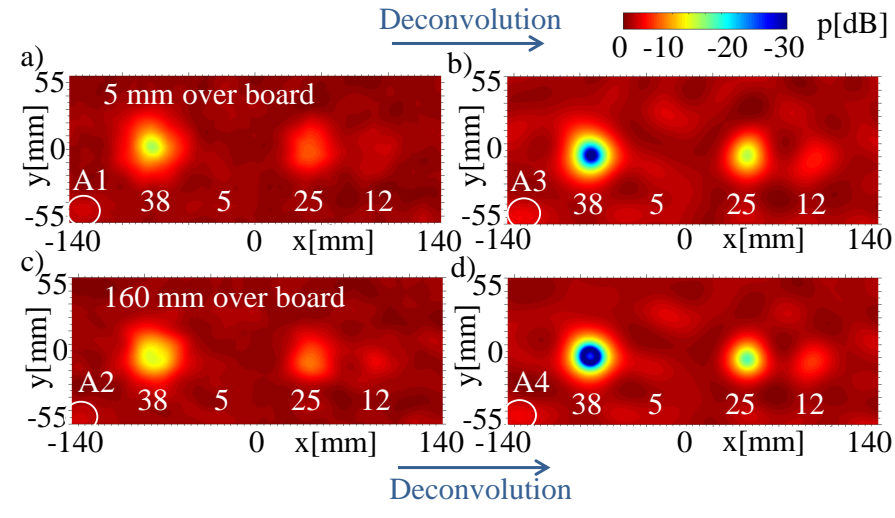

Fig. 7. ACU pitch-catch measurements of Medium Density Fiberboards (MDF). a) Measurement $5 \mathrm{~mm}$ over the board. b) C-scan after the deconvolution process. c) Measurement $160 \mathrm{~mm}$ over the board. d) C-scan after the deconvolution process.

can be in general particularized to detect inner composite defects. We analyzed the performance in this case by gluing paper snippets with adhesive tape on the receiver side of the board. In order to compare it with measurement with flaws on the transmitter side, the panel was rotated by $180^{\circ}$ around the $\mathrm{x}$-axis (first flaws on top (RX side) then on bottom (TX side)). Afterwards the measurement of the sound field by the point receiver was repeated. For ACU-TR at transmitter side, an additional ACU backwards re-radiation step is performed through the composite panel. This is achieved in two steps. First, ACU re-radiation is performed on the receiver side to compensate for diffraction in air along the $160 \mathrm{~mm}$ ACU RXcomposite path. Then the calculated transient signals are used as input to a second re-radiation step, where re-radiation is performed through the composite. In this case the re-radiation uses the speed of sound of the particleboard composite.

For re-radiation calculations, all algorithms described above were implemented in Matlab (The Mathworks Inc., Natick, MA, USA). The speed of sound in the panel $c_{1}$ was estimated as $750 \mathrm{~m} / \mathrm{s}$ from relative time measurements in transmission direction [66].

\section{RESUlTS}

\section{A. ACU pitch-catch measurements}

Fig.77 shows pitch catch measurement results for the 22 $\mathrm{mm}$ thick MDF-board. Fig.7 $7 \mathrm{l}$ and 7 display experimental Cscans of measurements $5 \mathrm{~mm}$ and $160 \mathrm{~mm}$ above the board, respectively. In both measurements, the defects with a diameter of 25 and $38 \mathrm{~mm}$ are visible. The defects of diameter $12 \mathrm{~mm}$ are hardly distinguishable from material inhomogeneities (see A1/A2 in Fig. 7 $7 \mathrm{a}$ and $7 \mathrm{f}$ ). No contrast is observed for the $5 \mathrm{~mm}$ diameter defects. The main resolution limiting effect is here the large receiver transducer area, and the image quality does not significantly improve at shorter sample-receive distances (5 mm instead of $160 \mathrm{~mm}$ ). The outputs of the deconvolution processing are shown in Fig.7b and 77. $\omega_{0}=0.08$ and $D\left(\omega_{0}\right) / D(0)=50$ were empirically found to be good tradeoff values for Eq. (16) between image sharpness and noise amplification. The deconvolution filter improves the contrast 

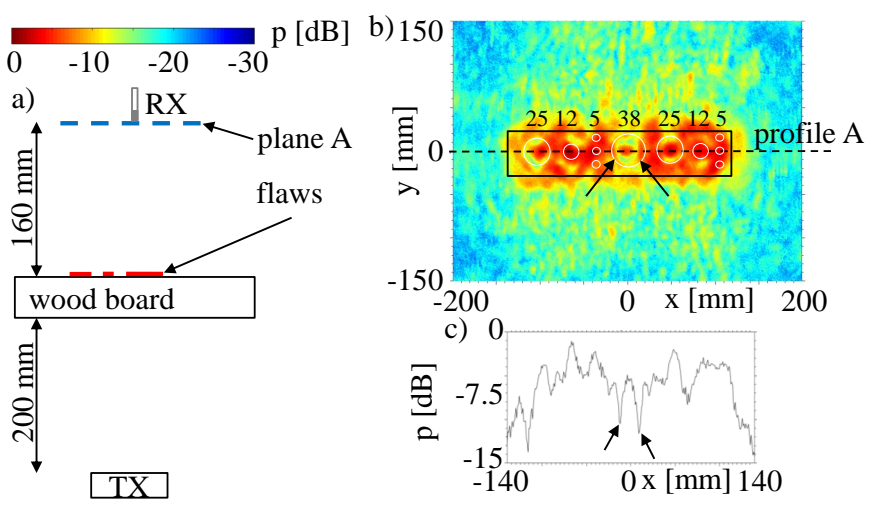

Fig. 8. Pressure field $160 \mathrm{~mm}$ above the medium fiberboard (MDF) composite. a) Experimental setup and plane position, the sound field is acquired in plane A. Flaws are located on top of the board. b) C-scan at plane A. c) Pressure profile. The transmission amplitude at defect regions shows a ring pattern, with an amplitude decrease only at the defect edges (arrows in b and c).

for the $38 \mathrm{~mm}$ and $25 \mathrm{~mm}$ diameter defects by up to 20 $\mathrm{dB}$, allowing for a better visualization. However, the $12 \mathrm{~mm}$ and $5 \mathrm{~mm}$ defects are still not resolved from the background variability (see A3/A4 in Fig.7p and 7p). Thus, although deconvolution improves the defect contrast down to the pitch catch detection limit, in practice it does not significantly expand this limit to significantly smaller defect sizes.

\section{B. ACU Time-reversal measurements}

ACU TR measurements based on the setup of Fig.6 are schematically summarized in Fig.83. The input data for the re-radiation method was plane $A$, which was located $160 \mathrm{~mm}$ above the MDF board and represents the receiver distance in an equivalent industrial application [67]. The sound pressure distribution as measured by the microphone is shown as a C-scan in Fig. 8 p. The delamination defects with a diameter of 25 and $38 \mathrm{~mm}$ show a visible amplitude contrast, but the delamination contour cannot be identified. For delaminations with a diameter of $12 \mathrm{~mm}$, only small amplitude variations can be detected and the delaminations with a diameter of 5 $\mathrm{mm}$ are not recognizable. Ring structures are observed for all detectable effects, with a drop of the pressure field at the defect edges and an increase of transmission signal in the central part of the defects (see arrows in Fig. 8b). These findings are confirmed by the profile on the y-axis (Fig.86). The maximum drop is only $-12.1 \mathrm{~dB}$ for the largest flaws and no change in amplitude can be detected for the smallest flaws.

Based on the measurement of plane A, the sound pressure distribution was calculated $5 \mathrm{~mm}$ above the board using the ACU-TR method (Fig.99). To validate the calculations, the calculated ACU-TR C-scan in this plane was compared to a direct measurement with the microphone (Fig.9p). A striking improvement of image resolution is achieved, with all defects clearly resolved in this case. Both measured (Fig.99) and calculated (Fig.99) C-scans show a very good agreement, which is quantitatively visualized on the $\mathrm{x}$-axis profile (Fig.98). Not only the defect profiles, but also natural material inhomogeneity are consistently visualized in both

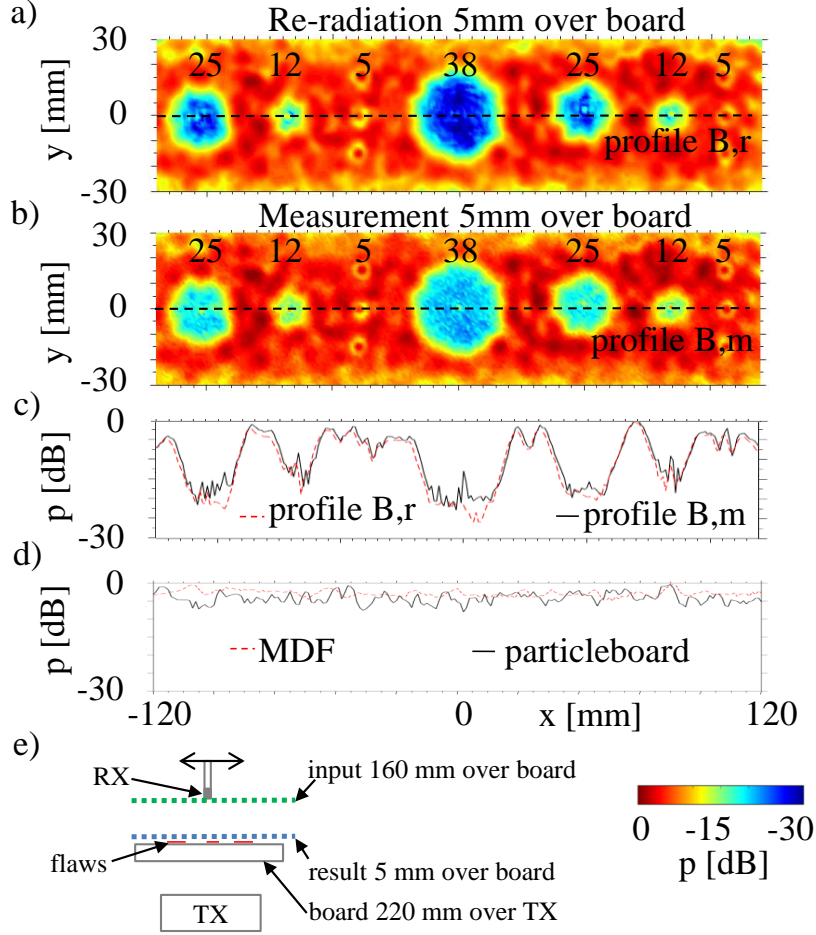

Fig. 9. Comparison between direct microphone measurement and ACU-TR image for imaging at a plane $5 \mathrm{~mm}$ above the MDF board and flaws on the top-side of the board. a) C-scan of the ACU-TR result based on a measurement $160 \mathrm{~mm}$ above the board. b) C-scan of direct microphone measurement $5 \mathrm{~mm}$ over the board. c) Pressure profile at $\mathrm{y}=0 \mathrm{~mm}$ for the re-radiation (profile $\mathrm{B}, \mathrm{r}$ ) and the measurement result (profile B,m). d) Pressure profiles in representative defect-free regions for both MDF and PB. e) Measurement schematic. Defect position plane is indicated in the with red dashed lines, calculated C-scan plane with blue dashed line and the input plane for the re-radiation with green dashed line.

measured and calculated pressure fields. Remarkably, the amplitude contrast for large defects $(38 \mathrm{~mm}, 25 \mathrm{~mm})$ in the calculated ACU-TR images (Fig.9a) is even $6.2 \mathrm{~dB}$ higher than for the directly measured microphone field (Fig.9p). This is interpreted considering that the directly measured pressure fields show additional noise and spurious signal sources (for instance, noise floor of the microphone, reverberations in microphone capsule, phantom echoes from previous transmit cycles), while the filtered summation of the ACU-TR computation effectively acts as a noise reduction filter.

In contrast to the far-field microphone measurement (Fig. 8p), all defects are detected in the ACU-TR C-scan (Fig.97). Even the smallest delaminations (diameter $5 \mathrm{~mm}$ ) are visible. The smallest $5 \mathrm{~mm}$ defects still show the ring pattern previously observed for larger defects in Fig. 8f. The transmission contrast at defects in Fig.9r) is significantly higher than in the profile at plane A (Fig. 8c). The smallest delaminations are detected with an amplitude contrast of almost $10 \mathrm{~dB}$ and the contrast for larger flaws exceeds $20 \mathrm{~dB}$. Fig.9 $9 \mathrm{~d}$ shows the amplitude profile for a defect free MDF and particle board along the $\mathrm{x}$-axis. For defect-free MDF, the standard deviation is $0.85 \mathrm{~dB}$ and the difference between the maximum value $(-0.33 \mathrm{~dB})$ and the minimum value $(-4.95 \mathrm{~dB})$ is $4.62 \mathrm{~dB}$. For the particle board, the standard deviation of $1.54 \mathrm{~dB}$ is 
a)

b)
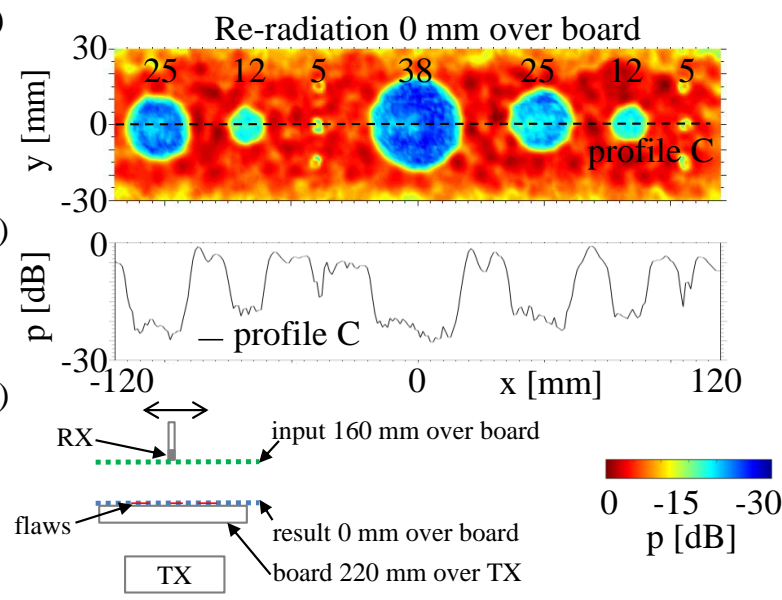

Fig. 10. Results of the ACU-TR method for the calculation of the pressure distribution directly on the board surface. a) C-scan. b) Pressure profile. c) Measurement schematic. Defect position plane is indicated in the with red dashed lines, calculated C-scan plane with blue dashed line and input plane for the re-radiation with green dashed line.

almost twice as high and the difference is $7.54 \mathrm{~dB}$ (max. $-0.51 \mathrm{~dB}$, min. $-8.05 \mathrm{~dB}$ ). So for the detection of defects it is necessary that the amplitude drop is above a limit of about -8 $\mathrm{dB}$, in order to avoid overlaps with natural material variability.

Fig. 10 shows the results of $\mathrm{C}$-scan calculations directly on the MDF board surface, exactly at the defect plane location. This plane is otherwise not directly accessible without damage to the microphone, and therefore only ACU-TR results are available. Compared to Fig.9, the resolution could be further improved and the contours of the delaminations can be seen even more clearly, especially for the smallest $5 \mathrm{~mm}$ defects, where the ring pattern effects are alleviated (Fig. 10 a). This can also be recognized by the steeper slope in the profile at the delamination edges (Fig. $10 \mathrm{p}$ ). The amplitude contrast as well improves for the smaller size $(5,12$ and $25 \mathrm{~mm})$ defects. As for the largest defects $(38 \mathrm{~mm})$, the contrast does not increase above $25 \mathrm{~dB}$. A reason for this limit is the $30 \mathrm{~dB}$ cut-off amplitude threshold for the input signals in the ACUTR calculation. It can be expected that signal variations below this threshold cannot be resolved.

An improved visualization of the wave propagation effects associated to the defect resolution limits is shown in Fig. 11 , where the re-radiated fields are visualized in a xz-cross section between the panel and receiver plane corresponding to the radiation plane $(y=0)$. The increase of amplitude contrast is clearly visible towards the distance corresponding to the flaw plane $(\mathrm{z}=0)$. With increasing distance from this plane, diffraction effects lead to a significant decrease in the minimum detectable delamination size. The previously observed ring artifacts are associated to symmetric side lobes, which are caused by diffraction at both defect edges. These signals interfere at the center of the defect region, leading to an apparent transmission increase (arrows in Fig. 11). The amplitude contrast in function of the measurement method and the defect diameter is summarized in Table[ ACU-TR clearly outperforms conventional pitch-catch methods (with

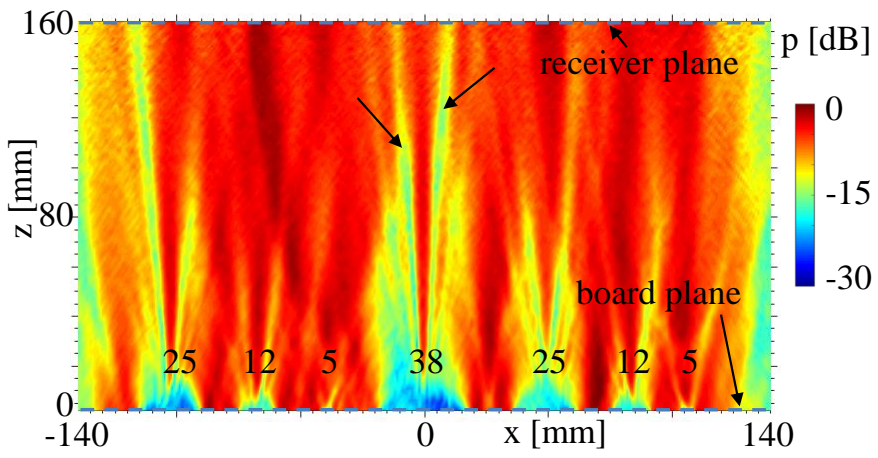

Fig. 11. Peak sound pressure distribution between the MDF board and the receiver, calculated with $\mathrm{ACU}-\mathrm{TR}$ form a B-scan measured at $\mathrm{z}=160 \mathrm{~mm}$. The arrows mark diffraction side lobes, which lead to the ring patterns observed in Fig. 8 p.

and without deconvolution) for defect sizes $<25 \mathrm{~mm}$, and allows detection of $5 \mathrm{~mm}$ diameter defects not visible with the latter. The natural amplitude variability in defect-free material is slightly larger in the pitch-catch setup than in ACUTR. This noise reduction may be associated to the averaging effect of the receiver transducer of the pitch-catch setup. For large delaminations $(38 \mathrm{~mm})$, the contrast of pitch-catch after deconvolution is also larger than in ACU-TR. This may be explained by the edge enhancing effect of the deconvolution filters and the cut-off value defined in ACU-TR. Both of these observations do not have a practical impact on defect detectability.

TABLE I

AMPLITUDE CONTRAST FOR DIFFERENT ACU METHODS AND DEFECT SIZES. DEFECT-FREE CORRESPONDS TO THE NATURAL MATERIAL VARIABILITY. THE BEST-CASE VALUES ARE HIGHLIGHTED. PITCH-CATCH (P.-C.); Deconvolution (De.); Microphone measurement (Mic).

\begin{tabular}{c|c|c|c|c}
\hline $\begin{array}{c}\text { Delamina- } \\
\text { tion size }\end{array}$ & $\begin{array}{c}\text { P.-C. } \\
160 \mathrm{~mm}\end{array}$ & $\begin{array}{c}\text { P.-C. } \\
5 \mathrm{~mm}\end{array}$ & $\begin{array}{c}\text { P.-C.+De. } \\
160 \mathrm{~mm}\end{array}$ & $\begin{array}{c}\text { P.-C.+De. } \\
5 \mathrm{~mm}\end{array}$ \\
\hline \hline $38 \mathrm{~mm}$ & $11.1 \mathrm{~dB}$ & $12.3 \mathrm{~dB}$ & $29.7 \mathrm{~dB}$ & $\mathbf{3 2 . 1} \mathrm{dB}$ \\
$25 \mathrm{~mm}$ & $6.3 \mathrm{~dB}$ & $6.4 \mathrm{~dB}$ & $16.2 \mathrm{~dB}$ & $14.2 \mathrm{~dB}$ \\
$12 \mathrm{~mm}$ & $3.4 \mathrm{~dB}$ & $2.9 \mathrm{~dB}$ & $5.4 \mathrm{~dB}$ & $4.6 \mathrm{~dB}$ \\
$5 \mathrm{~mm}$ & - & - & - & - \\
defect-free & $\mathbf{2 . 3} \mathrm{dB}$ & $2.4 \mathrm{~dB}$ & $3.7 \mathrm{~dB}$ & $3.6 \mathrm{~dB}$ \\
\hline Delamina- & Mic. & Mic. & ACU-TR & ACU-TR \\
tion size & $160 \mathrm{~mm}$ & $5 \mathrm{~mm}$ & $5 \mathrm{~mm}$ & $0 \mathrm{~mm}$ \\
\hline \hline $38 \mathrm{~mm}$ & $12.1 \mathrm{~dB}$ & $20.3 \mathrm{~dB}$ & $24.1 \mathrm{~dB}$ & $24.7 \mathrm{~dB}$ \\
$25 \mathrm{~mm}$ & $8.2 \mathrm{~dB}$ & $18.2 \mathrm{~dB}$ & $20.2 \mathrm{~dB}$ & $\mathbf{2 2 . 5} \mathrm{dB}$ \\
$12 \mathrm{~mm}$ & $4.7 \mathrm{~dB}$ & $15.4 \mathrm{~dB}$ & $15.5 \mathrm{~dB}$ & $\mathbf{1 7 . 3} \mathrm{dB}$ \\
$5 \mathrm{~mm}$ & - & $8.1 \mathrm{~dB}$ & $8.2 \mathrm{~dB}$ & $\mathbf{1 5 . 1} \mathrm{dB}$ \\
defect-free & $4.2 \mathrm{~dB}$ & $4.6 \mathrm{~dB}$ & $4.5 \mathrm{~dB}$ & $4.9 \mathrm{~dB}$ \\
\hline
\end{tabular}

\section{ACU time-reversal through wood composite board}

In the previous examples, defects were introduced at the sample surface on the receiver side, so that re-radiation of ACU sound fields were only performed through air. The most extreme detection case with respect to the ACU-TR procedure is for flaws located at the transmitter side of the panel, where re-radiation through the full panel thickness is necessary. Fig.12 shows a C-scan calculated with ACUTR at sample surface with flaws on the receiver side. The 


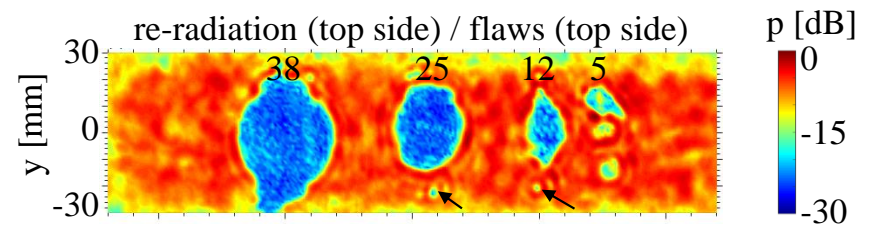

Fig. 12. Results of the ACU-TR method (input plane $160 \mathrm{~mm}$ above the board, same setup as Fig 10 for the calculation of the pressure distribution when the flaws are on the receiver side of a particleboard (PB) sample. Sub-wavelength defect resolution is achieved, allowing detection of small air bubbles on the sample surface (two air bubbles of respective diameters 3 and $2 \mathrm{~mm}$ are marked with arrows).

lateral resolution of Fig 12 is similar to the experiments in the medium fiberboard panel (MDF) (Fig. 10). As a result of the larger wood particles in the PB sample, stronger scattering effects occur in $\mathrm{PB}$ and the signal to noise ratio of the $\mathrm{C}$ scan is slightly reduced with respect to MDF. Additionally, irregular defect boundaries are observed in Fig. 12. These were introduced by the adhesive tape with which the paper strips were fixed to the board in the PB case. The tape also created additional air gaps between the tape and the board surface, especially at the edge of the paper snippets. For this reason, the contours of the delaminations are not circular as in Fig. 10. By using the ACU-TR method it is possible to visualize small air bubbles between tape and board bubbles (marked with arrows) at the edge of the defined defects down to a diameter of $2 \mathrm{~mm}$ (see Fig. 12). Considering that the wavelength in air is approximately $3.3 \mathrm{~mm}$, this experimentally shows that ACU-TR can invert defects with sub-wavelength resolution. We further analysed the transmission contrast within the air bubbles and observed a transmission drop of $15 \mathrm{~dB}$ for $3 \mathrm{~mm}$ air bubbles and $13 \mathrm{~dB}$ for $2 \mathrm{~mm}$ air bubbles.

Fig. 13 shows the application of the ACU-TR method to image defects on the transmitter side of the board. Calculated pressure distributions are shown at the receiver side (upper part) and transmitter side (bottom part) of the PB panel, where flaws are located at the transmitter side. In comparison to Fig. 12, a smeared image is observed when ACU-TR C-scans are calculated on the receiver side (Fig. 13 a), which in this case does not correspond to the defect plane.

Defect resolution significantly improves when C-scans are calculated on the transmitter side (Fig.13p). In this case, even if strong contrast variations are observed within the defects, they can at least be delineated by eye from their outer contours, which show circular patterns. Additional postprocessing or machine learning algorithms may be required to separate these geometric defect patterns from the background contrast variability. Defect sensitivity is degraded with respect to Fig.12a, with contrast going down to $-20 \mathrm{~dB}$ for the 38 and $25 \mathrm{~mm}$ diameter defects, and even lower $(-12 \mathrm{~dB})$ for the 5 $\mathrm{mm}$ defects. The reconstructed defects show ring patterns as in Fig. 8 p. While the contour of the defects is clearly visible, the inner part shows high amplitude values. This is not surprising, since we are using a simplified model based on a single speed of sound value to describe the wave diffraction into the heterogeneous anisotropic particleboard. We hypothesize that there are some parts of the pressure field which, due

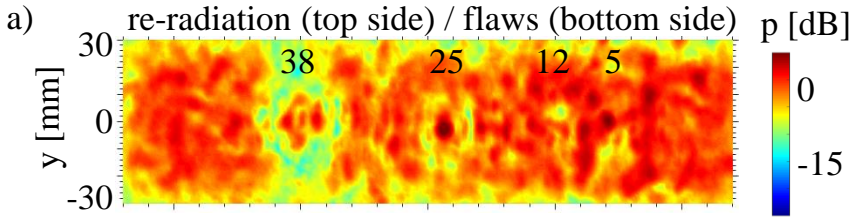

b) re-radiation (bottom side) / flaws (bottom side) -30

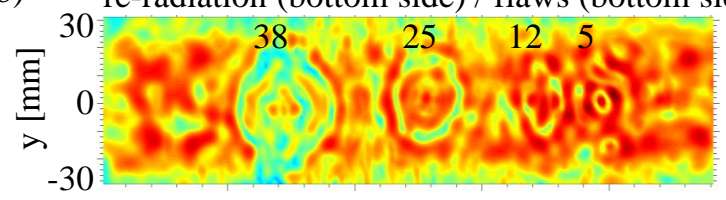

c) re-radiation (bottom side) / flaws (bottom side)

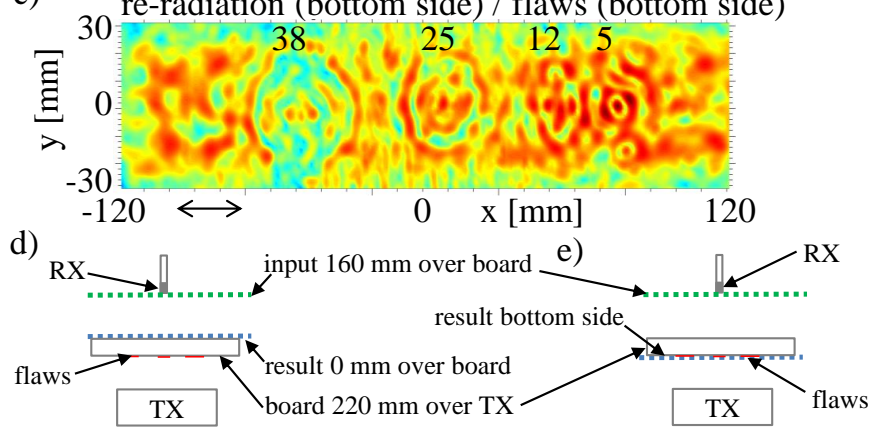

Fig. 13. Results of the ACU-TR method (input plane $160 \mathrm{~mm}$ above the board) for the calculation of the pressure distribution at different planes of the particleboard (PB) sample when the defects are on the transmitter side. a) Sound pressure over the board with flaw on the opposite side (C-scan plane not equal to flaw plane). b) Sound pressure on the lower side of the board (C-scan plane equal to flaw plane) calculated with Eq. 12). Although a visible improvement of lateral resolution is achieved between Fig. 13 a and Fig. 13 p, the resolution and defect contrast is degraded by the re-radiation through the PB particleboard panel. c) Sound pressure on the bottom side of the board calculated with simplified expression Eq. 10. The differences with Fig. 13p are negligible. d) Setup for a). e) Setup for b) and c).

to mode conversion and scattering, are not accounted for in our model. For instance, Sanabria et al. [68] found significant shear-wave conversion effects in solid wood boards at slanted ACU incidence.

Furthermore, the optimum defect delineation was in this case empirically found using for ACU-TR calculations a lower thickness value $(14 \mathrm{~mm})$ than the exact board thickness $(22 \mathrm{~mm})$, where defects are located. The anisotropy of the sound field maybe a cause for this observation. The speed of sound in $\mathrm{PB}$ is known to be at least twice larger inplane within the composite $(1500-2000 \mathrm{~m} / \mathrm{s})$ than across the plane (here $750 \mathrm{~m} / \mathrm{s})$ [8], [26]. Using a single isotropic speed of sound value in all directions introduces a simplification. It is therefore remarkable that $5 \mathrm{~mm}$ defects, well-below the estimated in-plane wavelength of the composite (13-17 mm), are still showing recognizable patterns in the images.

Comparing calculations accounting for the transmission coefficient at the air-sample interface (Eq. [12), as shown in Fig. 13 , with C-scans calculated with the simplified expression Eq. (10) (Fig.13k) does not shown significant differences in the C-scan (uncertainty $<20 \mathrm{~dB}$ ), so this does not seem to play a mayor difference in the re-radiation performance. 


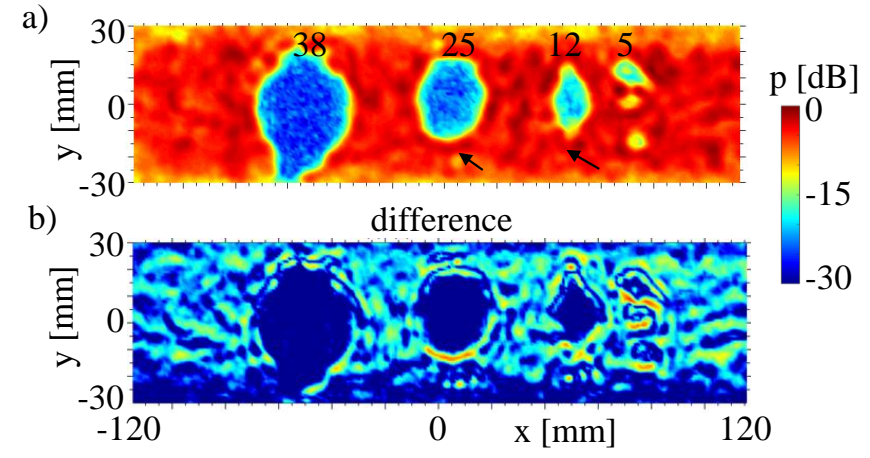

Fig. 14. Results of the ACU-TR method (setup as in Fig. 12] using twodimensional Fourier transforms Eq. (11) for re-radiation. a) Pressure field over the board. b) Difference image between Fig. 14 und Fig.12

\section{Computational optimizations}

The computation complexity for a re-radiation between two planes (each represented by an $N \times M$ grid) is $N^{2} M^{2}$. Using the fast Fourier approach the complexity reduces to $N \log (N) M \log (M)$, which for large grids is faster by one or two orders of magnitudes. The calculation time for the results shown in Fig. 10 could be decreased from 42 min to less than 2 min using a $3.3 \mathrm{GHz}$ CPU and $32 \mathrm{~GB}$ RAM. By using faster computational architectures, for instance, Graphical Processing Units (GPUs) and by considering the high parallelization of Eq. (11), a real time implementation of the computation is realistic. For the reconstruction of the pulsed waveform signals based on the two-dimensional Fourier transforms a frequency range of $60 \mathrm{kHz}-240 \mathrm{kHz}$ in steps of $1 \mathrm{kHz}$ was used.

The two-dimensional Fourier method shows slight tradeoffs between significant computational improvement and C-scan resolution (Fig. 14). Fig.14a shows an example of the Fourier calculation method to the example of Fig. 10. In this case, air droplets with a diameter of $3 \mathrm{~mm}$ still show an amplitude contrast of $-11 \mathrm{~dB}$, but the $2 \mathrm{~mm}$ droplets are no longer recognizable (illustrated with arrows). This effect can be minimized by performing the calculations with sampling of the entire relevant wavelength range. However, this increases the number of target frequencies and computation time for the reconstruction based on the two-dimensional Fourier transforms, with only a minor improvement in image quality. Overall, the two-dimensional Fourier optimization provides a good trade-off between high-resolution defect imaging and short data evaluation time.

\section{DiscuSSION AND CONCLUSION}

Non-destructive imaging is becoming increasingly important for bonding quality assessment of composite materials, which find increasing use in structural applications. ACU measurements provide a full-field imaging modality with high sensitivity to defect detection and delineation. The classic ACU pitch-catch setup is already widely used in industry. However, for thick heterogeneous composites, large size transducers with respect to the wavelength in air are presently necessary to achieve sufficient signal transmission through the samples.
In the presented experiments, the transducer diameter $D_{\mathrm{T}}$ to wavelength $\lambda$ ratio is $D_{\mathrm{T}} / \lambda=50 \mathrm{~mm} / 3.3 \mathrm{~mm}=15.2$. With planar (not focused) transducers and wood composite panels, defects can only be reliably detected down to half of the transducer diameter size, with the performance worsening for thicker composites materials and large sample-receiver distances. Deconvolution filters are post-processing algorithms, which are typically applied to the final amplitude images (Cscans). Deconvolution provides an edge detector filter, which somewhat enhances the apparent signal to noise ratio of the $\mathrm{C}$-scan images. However, this method does not significantly improve the detectability limit to smaller defect sizes. The depth localization of the defect plane within the composite is also not accessible.

In order to improve defect lateral resolution, a novel computational method has been introduced, ACU Time Reversal (ACU-TR). This method is based on acoustic holography and allows the projection of the measured ultrasound fields from an arbitrary measurement plane to an arbitrary defect plane. The ACU-TR method uses both the phase and amplitude information over the full ultrasound signal spectrum, and achieves compensation of ultrasound beam diffraction and refraction effects, thereby significantly improving the resolution of defect delineation. In particular, in our experiments we showed that the detectability limit for ACU pitch catch setup was around $20 \mathrm{~mm}$ for a $50 \mathrm{~mm}$ diameter transducer, whereas defects of $5 \mathrm{~mm}$ could be sharply delineated in the same test samples with ACU-TR. We incidentally observed that air droplets of $2 \mathrm{~mm}$ diameter could be as well detected with good contrast with the ACU-TR method, thus providing sub-wavelength resolution. The observation of sub-wavelength resolution is supported by the theoretical background of acoustic holography, which accounts for near field inversion of the pressure field [43]-[45], [69], [70]. However, considering the measurement uncertainties and discretization limits of the practical ACU-TR implementation, it is remarkable that sub-wavelength resolution is experimentally achievable with measurements acquired in the far field at $160 \mathrm{~mm}$ from the defect plane. Since our measurements were performed in realistic heterogeneous wood composite panels, we can also conclude that the complexity of the re-radiated pressure fields does not significantly degrade the performance of the method, as long as the wave propagation physics can be accurately modeled in the re-radiated media. This is in line with previous time reversal works, in which pressure field distributions were successfully reconstructed across complex scattering media [71], [72]. We have observed that, even when using a simplified acoustic model to describe re-radiation through anisotropic PB samples, defects down to $5 \mathrm{~mm}$ can be detected, which are smaller than the wavelength in the PB sample. Deviations from the actual wave propagation physics degrade the defect contrast sensitivity. Since mode-conversion, anisotropic diffraction and scattering phenomena are expected for wood-based materials [68], more sophisticated holography models are still necessary to optimize ACU-TR performance for re-radiation in inner planes of such composites. However, the current ACU-TR model is able to accurately resolve delaminations at the composite surfaces, which covers the most usual 
bonding quality assessment scenario in the wood composite panel production lines. We identified some upper limits to the amplitude contrast at delamination defects in ACU-TR reconstructions, which was reaching in our experiments a bottom line of $25 \mathrm{~dB}$. It is known that large air gaps of infinity lateral size lead to an amplitude contrast up to 50 $\mathrm{dB}$ [18]. A reason for the observed reduced contrast is the finite amplitude threshold for the input signals in the ACUTR calculation. Currently the limits of the rectangular scanning area are defined by a minimum $30 \mathrm{~dB}$ decrease in relation to the maximum pressure. The maximum pressure is obtained at a defect-free region, and pressure profiles are drawn in $\mathrm{x}$ and $y$ axes to identify the limits of the scanning area. Therefore, it is expected that signal amplitudes below this threshold cannot be resolved. Nevetherless, it is remarkable that the amplitude contrast obtained with ACU-TR (Fig.9a) was superior to the noise floor of direct microphone measurements (Fig.9p). This indicates that the computational approach has also an effective noise reduction effect.

The experiments with defects on alternating surfaces of the sample illustrated the applicability of the ACU-TR method in samples with multiple bonding planes located at different depths (Fig.12 and Fig.13. It was observed that the reradiation procedure achieves best defect lateral resolution when the C-scan plane corresponds to the flaw plane. In a composite with multiple bonding planes, this can be potentially used as an indication for localization of the faulty plane. If a list of possible defect planes is not available, for instance, for a solid component with cracks or air inclusions, it is feasible to estimate the defect depth by computing ACU-TR images at multiple depth values and by evaluating the image sharpness at each plane.

ACU-TR provides best defect contrast and resolution if the defects are flat and oriented parallel to the board plane and perpendicular to the ultrasound beam. For more complex defect geometries, more than one transmit projection at different insonification angles would be necessary in order to reconstruct the defect geometry. We are presently exploring extensions of the ACU-TR method to more complex tomographic configurations. The goal is to address complex defect geometries (e.g. defects slanted with respect to ACU beam, holes, etc.), and constrained inspection geometries, in which only single-sided or slanted access to the sample is available. For this purpose, more complex models of the material interfaces, including mode conversion phenomena, are necessary.

The scanned microphone emulates an array of point source receivers. Since the large-area planar transmitter covers the entire measurement region, the scanning effort reduces to the receiver The required measurement time is equivalent to the one of the pitch-catch setup. By substituting the microphone by a receiver array, the measurement data could be acquired within a single electric scan. Therefore, a full-field measurement method would be possible. With such an approach, inline measurement systems for delamination detection could potentially be realized.

At high production speeds, a line-shaped transmitter and a microphone array could potentially enable seamless real- time bonding quality monitoring with high spatial resolution. The measured sound fields can serve as an input for the re-radiation process, so that the sound distribution can be determined directly on the defect planes and small flaws can be detected. This offers the possibility to increase the distance between the sample to be measured and the ACU receiver. With sufficient separation between transducers and sample, hot and damping materials can be directly checked for defects during the production process. In this work, results in woodbased materials were shown, which were examined at low frequencies due to their high material thickness and damping. The ACU-TR method can also be applied for high frequency ranges. For instance, the re-radiation method has already been successfully applied to characterization of medical ultrasound imaging transducers in the megahertz range [57].

\section{ACKNOWLEDGMENT}

The experiments were performed at the Swiss Federal Laboratories for Materials Science and Technology (Empa, Dübendorf). Empa contributed to this study with their equipment.

\section{REFERENCES}

[1] C. Ralph, M. Silberstein, P. R. Thakre, and R. Singh, Mechanics of Composite and Multi-functional Materials, Volume 7. Cham: Springer International Publishing, 2016.

[2] K. K. Kar, Composite Materials. Berlin, Heidelberg: Springer Berlin Heidelberg, 2017

[3] R. Stössel, "Air-coupled ultrasound inspection as a new non-destructive testing tool for quality assurance," PhD Thesis, University Stuttgart, Stuttgart, 2003

[4] R. Y. Vun, "Ultrasonic characterization of engineering performance of oriented strandboard," PhD Thesis, Graduate School of the Louisiana State University, Berkeley, 2003.

[5] A. G. M. Hasenstab, "Integritätsprüfung von holz mit dem zerstörungsfreien ultraschallechoverfahren," PHD Thesis, Technical University Berlin, Berlin, 2006.

[6] U. Hilbers, H. Thoemen, J. Hasener, and A. Fruehwald, "Effects of panel density and particle type on the ultrasonic transmission through wood-based panels," Wood Science and Technology, vol. 46, no. 4, pp. 685-698, 2012.

[7] S. J. Sanabria, "Air-coupled ultrasound propagation and novel nondestructive bonding quality assessment of timber composites," PHD Thesis, Swiss Federal Institute of Technology Zurich, Zürich, 2012.

[8] T. Marhenke, J. Twiefel, J. Hasener, and J. Wallaschek, "Influences on the ultrasonic transmission behavior of wood based materials," Proceedings of the IEEE International Ultrasonics Symposium (IUS), Washingston DC, 2017.

[9] Y. Fang, L. Lin, H. Feng, Z. Lu, and G. W. Emms, "Review of the use of air-coupled ultrasonic technologies for nondestructive testing of wood and wood products," Computers and Electronics in Agriculture, vol. 137, pp. 79-87, 2017.

[10] M. C. Bhardwaj, D. O. Thompson, and D. E. Chimenti, "Phenomenally high transduction air/gas transducers for practical non-contact ultrasonic applications," Proceedings of AIP Conference, Chicago, pp. 920-927, 2009.

[11] P. Pallav, D. A. Hutchins, and T. H. Gan, "Air-coupled ultrasonic evaluation of food materials." Ultrasonics, vol. 49, pp. 244-253, 2009.

[12] E. Blomme, D. Bulcaen, T. Cool, F. Declercq, and P. Lust, "Air-coupled ultrasonic assessment of wood veneer," Physics Procedia, vol. 3, no. 1, pp. 193-200, 2010.

[13] L. J. Bond, C. Chiang, and C. M. Fortunko, "Absorption of ultrasonic waves in air at high frequencies (10-20 mhz)," The Journal of the Acoustical Society of America, vol. 92, no. 4, pp. 2006-2015, 1992.

[14] T. Gan, D. Hutchins, D. Billson, and D. Schindel, "High-resolution, air-coupled ultrasonic imaging of thin materials," IEEE Transactions on Ultrasonics Ferroelectrics and Frequency Control, vol. 50, no. 11, pp. 1516-1524, 2001. 
[15] R. Stoessel, N. Krohn, K. Pfleiderer, and G. Busse, "Air-coupled ultrasound inspection of various materials," Ultrasonics, vol. 40, pp. $159-163,2002$.

[16] T. Alvarez-Arenas, J. Camacho, and C. Fritsch, "Passive focusing techniques for piezoelectric air-coupled ultrasonic transducers," Ultrasonics, vol. 67, pp. 85-93, 2016.

[17] T. Potter, B. Ghaffari, and G. Mozurkewich, "Sub-wavelength resolution in air-coupled ultrasound images of spot welds," NDT \& E International, vol. 38 , no. 5, pp. 374-380, 2005.

[18] S. J. Sanabria, R. Furrer, J. Neuenschwander, P. Niemz, and U. Sennhauser, "Air-coupled ultrasound inspection of glued laminated timber," Holzforschung, vol. 65, no. 3, 2011.

[19] D. Algernon, B. Gräfe, F. Mielentz, B. Köhler, and F. Schubert, "Imaging of the elastic wave propagation in concrete using scanning techniques," Journal of Nondestructive Evaluation, vol. 27, no. 3, pp. 83-97, 2008.

[20] K. S. Hall and J. S. Popovics, "Air-coupled ultrasonic tomography of solids: 1 fundamental development," Smart Structures and Systems, vol. 17, no. 1, pp. 17-29, 2016.

[21] M. Kaczmarek, B. Piwakowski, and R. Drelich, "Noncontact ultrasonic nondestructive techniques: State of the art and their use in civil engineering," Journal of Infrastructure Systems, vol. 23, no. 1, p. B4016003, 2017.

[22] G. Revel, G. Pandarese, and A. Cavuto, "Advanced ultrasonic nondestructive testing for damage detection on thick and curved composite elements for constructions," Journal of Sandwich Structures \& Materials, vol. 15, no. 1, pp. 5-24, 2013.

[23] Fagus-GreCon Greten GmbH \& Co. KG, "Inline delamination detection with the ultrasonic measuring system," Technical Report, 2015.

[24] V. Bucur, Delamination in Wood, Wood Products and Wood-Based Composites, 1st ed. Dordrecht: Springer Science und Business Media, 2011.

[25] J. Bodig and B. A. Jayne, Mechanics of wood and wood composites, 1st ed. Malabar: Krieger Publishing, 1993.

[26] M. Dunky and P. Niemz, Holzwerkstoffe und Leime, 1st ed. Berlin, Heidelberg: Springer, 2002.

[27] U. Hilbers, "Untersuchungen zur luftangekoppelten ultraschallprüfung von holzwerkstoffen," PHD Thesis, University Hamburg, Hamburg, 2012.

[28] V. Bucur, Nondestructive characterization and imaging of wood. Springer Science \& Business Media, 2013.

[29] H. Sohn, D. Dutta, J. Y. Yang, M. DeSimio, S. Olson, and E. Swenson, "Automated detection of delamination and disbond from wavefield images obtained using a scanning laser vibrometer," Smart Materials and Structures, vol. 20, no. 4, pp. 1-11, 2011.

[30] W. J. Staszewski, B. C. Lee, and R. Traynor, "Fatigue crack detection in metallic structures with lamb waves and 3d laser vibrometry," Measurement Science and Technology, vol. 18, no. 3, p. 727, 2007.

[31] Y. Labyed and L. Huang, "Ultrasound time-reversal music imaging with diffraction and attenuation compensation," IEEE Transactions on Ultrasonics, Ferroelectrics and Frequency Control, vol. 59, no. 10, pp. 2186-2200, 2012.

[32] R. Bass, "Diffraction effects in the ultrasonic field of a piston source," The Journal of the Acoustical Society of America, vol. 30, no. 7, pp. $602-605,1958$.

[33] T. Marhenke, J. Neuenschwander, R. Furrer, J. Twiefel, J. Hasener, P. Niemz, and S. J. Sanabria, "Modeling of delamination detection utilizing air-coupled ultrasound in wood-based composites," $N D T \& E$ International, vol. 99, pp. 1-12, 2018.

[34] A. Hasenstab and M. Krause, "Luftultraschall und ultraschall-echotechnik an holz," DGZfP conference, Rostock, 2005.

[35] C. Yu, C. Zhang, and L. Xie, "A blind deconvolution approach to ultrasound imaging," IEEE Transactions on Ultrasonics, Ferroelectrics and Frequency Control, vol. 59, no. 2, pp. 271-280, 2012.

[36] P. Campisi, Blind Image Deconvolution: Theory and Applications, 1st ed. Hoboken: Taylor \& Francis Ltd, 2017.

[37] R. Rangarajan, C. V. Krishnamurthy, and K. Balasubramaniam, "Ultrasonic imaging using a computed point spread function," IEEE Transactions on Ultrasonics, Ferroelectrics and Frequency Control, vol. 55 , no. 2, pp. 451-464, 2008.

[38] A. Nowicki, J. Litniewski, and T. Kujawska, Acoustical Imaging, 1st ed. Dordrecht: Springer, 2012, vol. 31.

[39] G. Demoment, R. Reynaud, and A. Herment, "Range resolution improvement by a fast deconvolution method," Ultrasonic imaging, vol. 6, no. 4, pp. 435-451, 1984.

[40] E. E. Hundt and E. A. Trautenberg, "Digital processing of ultrasonic data by deconvolution," IEEE Transactions on Sonics and Ultrasonics, vol. 27 , no. 5, pp. 249-252, 1980.
[41] J. A. Jensen, "Deconvolution of ultrasound images," Ultrasonic imaging, vol. 14, no. 1, pp. 1-15, 1992

[42] S. J. Sanabria, T. Marhenke, R. Furrer, and J. Neuenschwander, "Calculation of volumetric sound field of pulsed air-coupled ultrasound transducers based on single plane measurements," IEEE Transactions on Ultrasonics, Ferroelectrics and Frequency Control, vol. 65, no. 1, pp. 72-84, 2018.

[43] R. Malkin, T. Todd, and D. Robert, "Quantitative imaging of acoustic reflection and interference," Proceedings of the 13th Anglo-French Physical Acoustics Conference, London, 2015.

[44] C. Trillo, A. F. Doval, S. Hernández-Montes, X. L. Deán-Ben, J. C. López-Vázquez, and J. L. Fernández, "Pulsed tv holography measurement and digital reconstruction of compression acoustic wave fields: Application to nondestructive testing of thick metallic samples," Measurement Science and Technology, vol. 22, no. 2, pp. 1-15, 2011.

[45] G. T. Clement and K. Hynynen, "Forward planar projection through layered media," IEEE Transactions on Ultrasonics, Ferroelectrics and Frequency Control, vol. 50, no. 12, pp. 1689-1698, 2003.

[46] M. Papadopoulos, "The reflexion and refraction of point-source fields," Proc. Roy. Soc. Ser. A, vol. 273, pp. 198-21, 1962.

[47] D.-L. Liu and R. C. Waag, "Propagation and backpropagation for ultrasonic wavefront design," IEEE Transactions on Ultrasonics, Ferroelectrics and Frequency Control, vol. 44, no. 1, pp. 1-13, 1997.

[48] X. Fan and K. Hynynen, "The effects of wave reflection and refraction at soft tissue interfaces during ultrasound hyperthermia treatments," $J$. Acoust. Soc. Am., vol. 91, pp. 1727-1736, 1992.

[49] J. Sun and K. Hynynen, "Focusing of therapeutic ultrasound through a human skull: A numerical study," The Journal of the Acoustical Society of America, vol. 104, p. 1705, 1998.

[50] X. Fan and K. Hynynen, "The effects of curved tissue layers on the power deposition patterns of therapeutic ultrasound beams," Med. Phys., vol. 21, pp. 25-34, 1994.

[51] L. W. Schmerr and S.-J. Song, Ultrasonic Nondestructive Evaluation Systems, 1st ed. Boston: Springer, 2007.

[52] M. Fink, "Time-reversed acoustics," Scientific American, vol. 281, no. 5, pp. 91-97, 1999.

[53] E. Hack and P. Zolliker, "Terahertz holography for imaging amplitude and phase objects," Optics express, vol. 22, no. 13, pp. 16079-16086, 2014.

[54] P. Zolliker and E. Hack, "Thz holography in reflection using a high resolution microbolometer array," Optics express, vol. 23, no. 9, pp. 10957-10967, 2015.

[55] N. Delen and B. Hooker, "Free-space beam propagation between arbitrarily oriented planes based on full diffraction theory: A fast fourier transform approach," Journal of the Optical Society of America A, vol. 15 , no. 4, p. $857,1998$.

[56] J. O. Smith, Mathematics of the discrete Fourier transform (DFT), 2nd ed. W3K Publishing, 2007.

[57] T. Marhenke, S. J. Sanabria, B. R. Chintada, R. Furrer, J. Neuenschwander, and O. Goksel, "Acoustic field characterization of medical array transducers based on unfocused transmits and single-plane hydrophone measurements," Sensors, vol. 19, no. 4, p. 863, 2019.

[58] E. Sekko, G. Thomas, and A. Boukrouche, "A deconvolution technique using optimal wiener filtering and regularization," Signal processing, vol. 72, no. 1, pp. 23-32, 1999.

[59] K. R. Castleman, Digital image processing, 2nd ed. Englewood Cliffs: Prentice Hall, 2003.

[60] S. Mallat, A wavelet tour of signal processing. Academic Press, 1999.

[61] S. V. Vaseghi, Advanced digital signal processing and noise reduction, 4th ed. Hoboken: Wiley, 2009.

[62] S. Gupta, R. C. Chauhan, and S. C. Sexana, "Wavelet-based statistical approach for speckle reduction in medical ultrasound images," Medical and Biological Engineering and computing, vol. 42, no. 2, pp. 189-192, 2004.

[63] S. Sudha, G. R. Suresh, and R. Sukanesh, "Speckle noise reduction in ultrasound images by wavelet thresholding based on weighted variance," International Journal of Computer Theory and Engineering, vol. 1, no. 1 , p. 7, 2009

[64] F. Simonetti, "Multiple scattering: The key to unravel the subwavelength world from the far-field pattern of a scattered wave," Physical review. E, Statistical, nonlinear, and soft matter physics, vol. 73, no. 3 Pt 2, p. 036619, 2006

[65] O. Cramer, "The variation of the specific heat ratio and the speed of sound in air with temperature, pressure, humidity, and $\operatorname{co} 2$ concentration," The Journal of the Acoustical Society of America, vol. 93, no. 5, pp. 2510-2516, 1993. 
[66] S. J. Sanabria, U. Hilbers, J. Neuenschwander, P. Niemz, U. Sennhauser, H. Thömen, and J. L. Wenker, "Modeling and prediction of density distribution and microstructure in particleboards from acoustic properties by correlation of non-contact high-resolution pulsed air-coupled ultrasound and x-ray images," Ultrasonics, vol. 53, no. 1, pp. 157-170, 2013.

[67] T. Marhenke, J. Twiefel, and J. Hasener, "Transiente ultraschallfeldvisualisierung von transmissionsvorgängen an platten auf basis der refraktovibrometrie," tm - Technisches Messen, vol. 83, no. 10, pp. 580592,2016

[68] S. J. Sanabria, R. Furrer, J. Neuenschwander, P. Niemz, and P. Schütz, "Analytical modeling, finite-difference simulation and experimental validation of air-coupled ultrasound beam refraction and damping through timber laminates, with application to non-destructive testing," Ultrasonics, vol. 63, pp. 65-85, 2015.

[69] S. K. Lehman and A. J. Devaney, "Transmission mode time-reversal super-resolution imaging," The Journal of the Acoustical Society of America, vol. 113, no. 5, pp. 2742-2753, 2003.

[70] J. D. Maynard, E. G. Williams, and Y. Lee, "Nearfield acoustic holography: I. theory of generalized holography and the development of nah," The Journal of the Acoustical Society of America, vol. 78, no. 4, pp. 1395-1413, 1985.

[71] C. Prada, E. Kerbrat, D. Cassereau, and M. Fink, "Time reversal techniques in ultrasonic nondestructive testing of scattering media," Inverse Problems, vol. 18, no. 6, pp. 1761-1773, 2002.

[72] N. Chakroun, M. A. Fink, and F. Wu, "Time reversal processing in ultrasonic nondestructive testing," IEEE transactions on ultrasonics, ferroelectrics, and frequency control, vol. 42, no. 6, pp. 1087-1098, 1995.

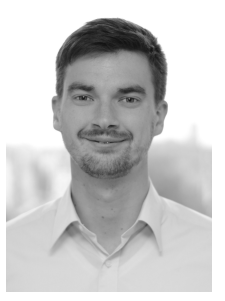

Torben Marhenke received the M.Sc. degree in mechanical engineering from Leibniz University Hannover, Hannover, Germany, in 2014. He has been pursuing the Ph.D. degree with Institute for Dynamics and Vibration Research, Leibniz University Hannover in cooperation with Fagus-GreCon Greten $\mathrm{GmbH}$ and Co. KG. Since 2019 he is with the Research and Development Department, Fagus-GreCon Greten GmbH and Co. KG, Alfeld, Germany. He is currently involved in NDE of wood-based materials with ultrasound.

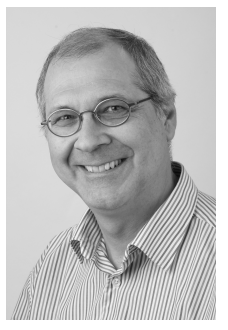

Jürg Neuenschwander received the M.Sc. degree in physics and the Ph.D. degree in natural sciences from the Swiss Federal Institute of Technology, ETH Zurich, Switzerland, in 1983 and 1988, respectively. In 1992, he joined the Swiss Federal Laboratories for Materials Science and Technology, Empa, Dbendorf, Switzerland, where he had finally a position as Senior Scientist in ultrasonics with the Transport at Nanoscale Interfaces Laboratory. Since 2018 he is retired.

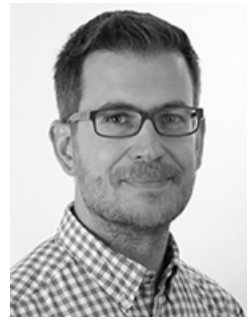

Roman Furrer received the Diploma FH in mechanical engineering from the Universities of Applied Sciences (HSR), Rapperswil, Switzerland, in 2007. He was with IPEK, an institute of HSR, as an Assistant, and then joined the Swiss Federal Laboratories for Materials Science and Technology, Empa, Duebendorf, Switzerland, in 2009. His current field of activity is CVD graphene growth and material research with ultrasonics, thermograpics and radiographics.

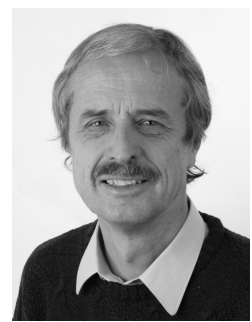

Peter Zolliker received the degree in physics from the Swiss Federal Institute of Technology, Zurich, and the Ph.D. degree in crystallography from the University of Geneva, Switzerland, in 1987. In 2003 , he joined the Media Technology Lab at the Swiss Federal Laboratories for Materials Testing and Research (Empa) and did research on imaging, image quality and psychophysics. In 2014 he changed to the Laboratory for Reliability Science and Technology at Empa which was renamed to Laboratory for Transport at Nanoscale Interfaces in 2017, where he heads the Measurement Technology Group.

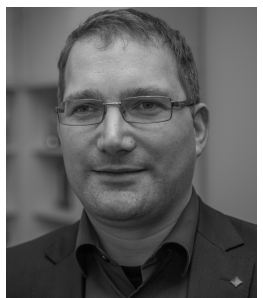

Jens Twiefel heads the research laboratory for piezoelectric and ultrasonic technology at the Institute of Dynamics and Vibration Research at Leibniz Universitt Hannover. His research focuses on the adaptation of high-performance ultrasonic technology to new applications and the improvement of the fundamental understanding of the influence of ultrasound on various production processes. A special focus lies on the holistic ultrasonic system from the electromechanical energy conversion to the mechanical vibration system up to the process.

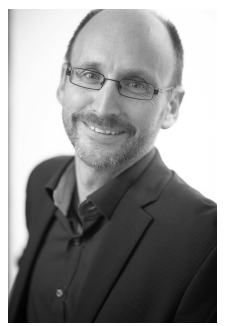

Jörg Hasener studied wood science and economy at the University of Hamburg. In 2004 he obtained his $\mathrm{PhD}$ at the University of Hamburg with a thesis on data-based prediction models in the production of wood-based materials. Since 2005 he has been working for Fagus-GreCon Greten GmbH \& Co KG in Alfeld. Here he initially held a leading position in measurement technology development for 8 years and has been responsible for innovation and patent management for 4 years. Since 2018 he is head of the Business Unit Measurement Technology.

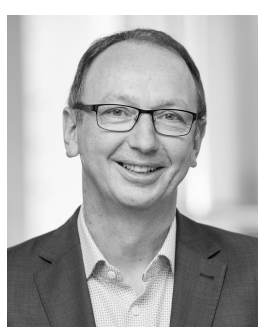

Jörg Wallaschek is professor of mechanics and director of the Institute of Dynamics and Vibration Research at the Leibniz Universitt Hannover, Germany. He holds a PhD and habilitation from the Technical University Darmstadt. His research interests include nonlinear dynamics, contact mechanics and friction as well as piezoelectric and ultrasonic technologies.

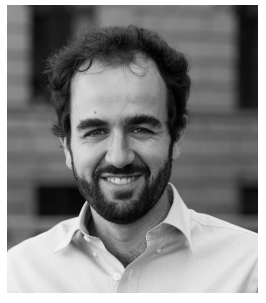

Sergio J Sanabria received the M.Sc. degree in telecommunications engineering from the University of the Basque Country, Bilbao, Spain, in 2007, and the Ph.D. degree from ETH Zurich, Switzerland, in 2012. From 2012 to 2014, he was a postdoc at the Institute for Building Materials and from 2014 to 2018 Senior Assistant at the Computer Vision Laboratory of ETH. Since 2018, he is pursuing his habilitation at the University Hospital Zurich, where he develops quantitative ultrasound methods for clinical diagnostics and material testing. 